\title{
Analysis of Modular-iterative Mixed Biosynthesis of Lankacidin by Heterologous Expression and Gene Fusion
}

\author{
Satoshi Tatsuno, Kenji Arakawa, Haruyasu Kinashi
}

Received: July 31, 2007 / Accepted: October 23, 2007

(C) Japan Antibiotics Research Association

\begin{abstract}
Lankacidin is a unique 17-membered macrocyclic antibiotic different from usual even-membered macrolides. Based on the gene organization of the lankacidin biosynthetic cluster coded on the linear plasmid pSLA2-L in Streptomyces rochei, we previously proposed a hypothesis of modular-iterative mixed polyketide biosynthesis for lankacidin. Two experimental evidences in this paper further strengthened this hypothesis. Heterologous expression of the lankacidin cluster ( $l k c A$ $l k c O$ ) in Streptomyces lividans resulted in lankacidinol A production, indicating that the gene cluster is sufficient for the synthesis of the lankacidin skeleton. In addition, a gene fusant of $l k c F$ and $l k c G$ produced lankacidin at a similar level to the parent strain, suggesting that an iterative function of the LkcF protein is unlikely. These results are consistent with the hypothesis that $\mathrm{LkcC}$ is used four times and $\mathrm{LkcA}, \mathrm{LkcF}$ and $\mathrm{LkcG}$ are used modularly to accomplish eight condensation reactions leading to the lankacidin skeleton.
\end{abstract}

Keywords antibiotic, polyketide synthase, Streptomyces, linear plasmid, biosynthesis, lankacidin

\section{Introduction}

Bacterial modular type-I polyketide synthases (PKSs) synthesize a variety of polyketide antibiotics such as macrolides, ansamycins, polyethers, and polyenes by sequential condensation reactions of short chain carboxylic

H. Kinashi (Corresponding author), S. Tatsuno, K. Arakawa: Department of Molecular Biotechnology, Graduate School of Advanced Sciences of Matter, Hiroshima University, HigashiHiroshima 739-8530, Japan, E-mail: kinashi@hiroshima-u.ac.jp acids [1]. Modular type-I PKSs are composed of modularly arranged sets of ketosynthase (KS), acyltransferase (AT), and acyl carrier protein (ACP) domains that are responsible for condensation reaction, the selection and transfer of extender units, and the retention of growing polyketide chains, respectively. In addition, modular PKSs contain modifying domains such as ketoreductase (KR), dehydratase (DH), and enoylreductase (ER), which give complex structural diversity to polyketide compounds. Thus, the domain organization in modular type-I PKSs has a strict colinear relationship with the order of biosynthetic steps.

However, metabolites without this relationship are sometimes produced by modular PKSs as minor components of fermentation. For example, 16-membered erythromycins were isolated from Saccharopolyspora erythraea, as a result of aberrant repeated use (termed PKS stuttering) of 6-deoxyerythronolide B synthases [2]. In contrast, ringcontracted epothilone derivatives were isolated as a result of PKS skipping in addition to ring-enlarged derivatives [3]. Different from such sporadic loss of colinear relationship, programmed iterative use of modular PKSs has been recently reported for stigmatellin in Stigmatella aurantiaca [4], borrelidin in Streptomyces parvulus [5], and aureothin in Streptomyces thioluteus [6]. In borrelidin biosynthesis, six modules catalyze eight rounds of chain elongation and modification, suggesting that module 5 (BorA5) is used three times [5].

Lankacidin C, a unique 17-membered macrocyclic antibiotic (1, Fig. 1) produced by Streptomyces rochei 7434AN4, is another example of iterative use of modular PKSs. Strain 7434AN4 carries three linear plasmids, pSLA2-L, -M, and -S [7, 8], and the complete sequencing of pSLA2-L together with extensive gene disruption experiments revealed that the lankacidin synthase $(l k c)$ 
gene cluster is located on pSLA2-L and spans $39 \mathrm{~kb}$ in size (Figs. 2 and 3A) [9, 10]. The $l k c$ cluster contains one nonribosomal peptide synthetase (NRPS)-PKS hybrid gene $(l k c A)$, three multidomain PKS genes $(l k c C, l k c F$, and $l k c G$ ), pyrroloquinoline quinone (PQQ) biosynthetic genes $(l k c K-l k c O)$, and an amine oxidase gene $(l k c E)$ [9]. In addition, the $l k c$ cluster contains discrete AT $(l k c D)$ and DH $(l k c B)$ genes, both of which act in trans in lankacidin biosynthesis.

The gene organization of the $l k c$ cluster raised two interesting questions. (i) How can five ketosyntase domains in the cluster accomplish eight condensation reactions

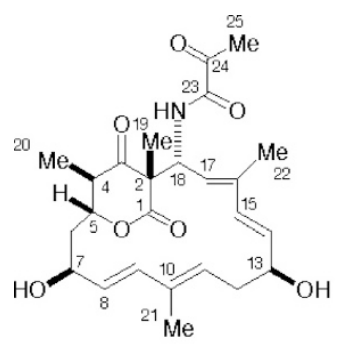

1

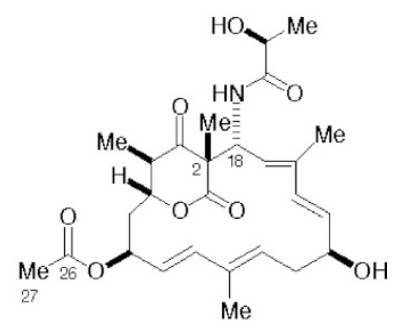

2
Fig. 1 Chemical structures of lankacidin C (1) and lankacidinol A (2). necessary for lankacidin synthesis. (ii) How is formed the carbon-carbon linkage between $\mathrm{C} 2$ and $\mathrm{C} 18$, which generates a unique 17-membered macrocyclic skeleton. We have already answered the second question [10]; namely, the amine oxidase (LkcE) converts an acyclic amide (C18N) intermediate (3, Fig. 2) to an imide $(\mathrm{C} 18=\mathrm{N})$, which then receives a nucleophilic attack by $\mathrm{C} 2$ to form the C2 C18 linkage in lankacidinol A (2, Fig. 1). To the first question, we proposed a modular-iterative mixed biosynthesis hypothesis (Fig. 2), where the LkcC protein functions iteratively and the remaining three PKS proteins (LkcA, LkcF and LkcG) function modularly. This hypothesis exactly agrees with the chemical structure of lankacidin; namely, four times use of $\mathrm{LkcC}$ extends a polyketide chain from $\mathrm{C} 14$ to $\mathrm{C} 7$, and two KR domains in $\mathrm{LkcF}$ reduce the ketone groups at $\mathrm{C} 7$ and $\mathrm{C} 5$ positions. The repeated use of the MT domain in $\mathrm{LkcC}$ could introduce four methyl groups at $\mathrm{C}-2,4,10,16$ positions. However, the following alternative possibilities could not be ruled out; (i) additional PKSs coded on pSLA2-L or on the chromosome might be involved, and (ii) other PKS modules ( $\mathrm{LkcF}$ and/or LkcG) might function iteratively.

To support the hypothesis of modular-iterative mixed polyketide biosynthesis for lankacidin, we carried out heterologous expression of the lkc cluster in Streptomyces

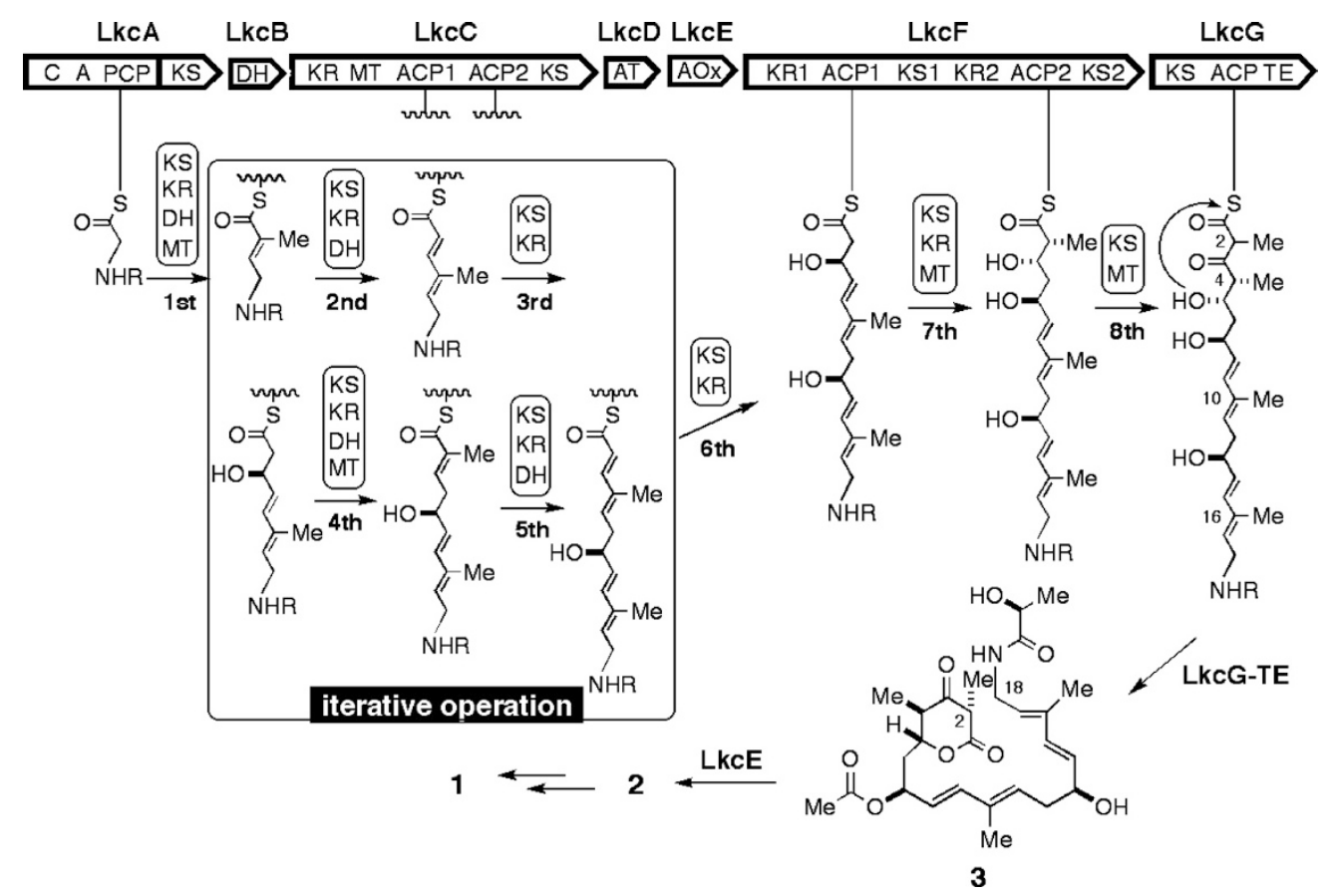

Fig. 2 Possible biosynthetic pathway of lankacidin.

$\mathrm{R}, \mathrm{CH}_{3}-\mathrm{CH}(\mathrm{OH}) \mathrm{C}(=\mathrm{O})-; \mathrm{C}$, condensation domain; $\mathrm{A}$, adenylation domain; $\mathrm{PCP}$, peptidyl carrier protein; KS, $\beta$-ketoacyl-ACP synthase; ACP, acyl carrier protein; KR, $\beta$-ketoacyl-ACP reductase; MT, $C$-methyltransferase; TE, thioesterase; AT, acyltransferase; $\mathrm{DH}, \beta$-hydroxy-acyl-ACP dehydratase; $\mathrm{AOx}$, amine oxidase. 


\section{A}

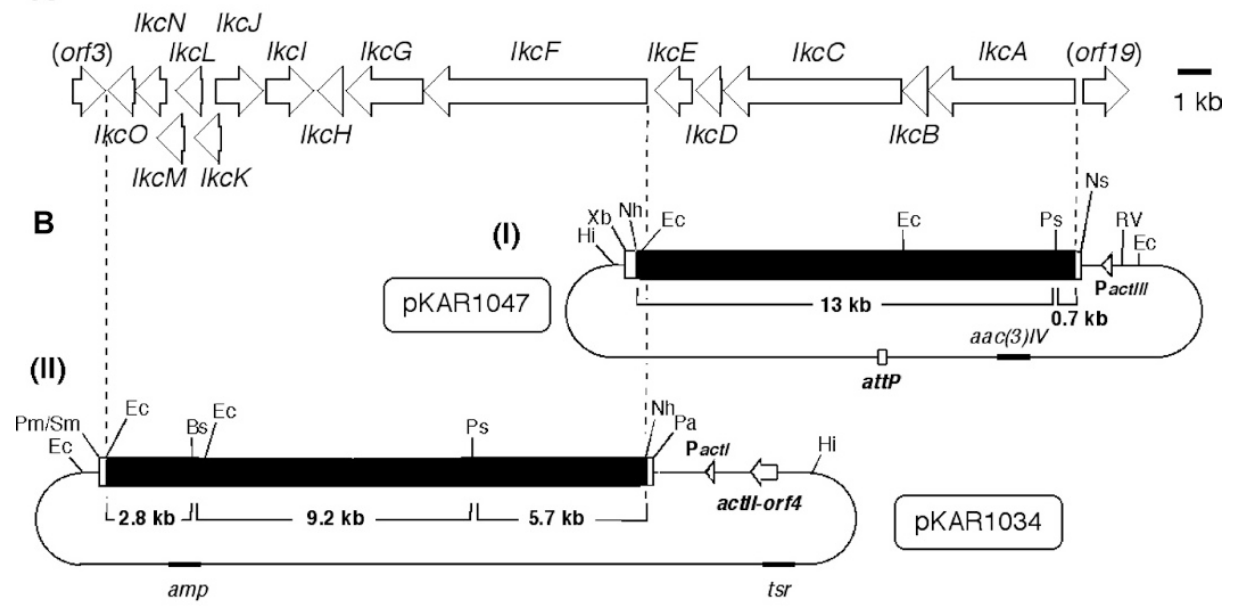

(III) S. lividans strain KA53 $(I k c A-I k c E)$

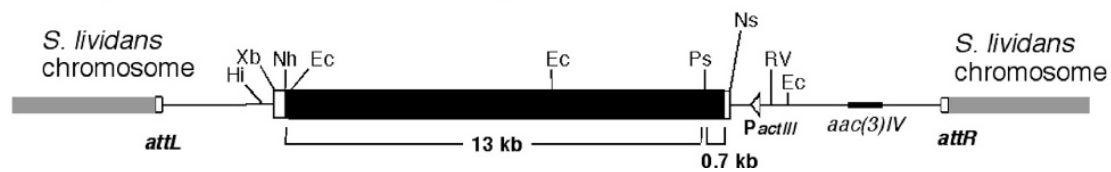

Fig. 3 Heterologous expression of the Ikc cluster.

(A) Gene organization of the Ikc cluster. (B) Cloning and integration of the Ikc subcluster. (I) pKAR1047 carrying IkcA-IkcE. (II) pKAR1034 carrying $I K c F-I k c O$. (III) $S$. lividans $K A 53$ in which the $I K c A-I k c E$ region was integrated into the chromosome at the attP site. actll-orf4, transcriptional activator gene; Pactl, act/ promoter; PactIII, act/ll promoter; attP, phage attachment site; aac(3)IV, apramycin resistance gene; amp, ampicillin resistance gene; tsr, thiostrepton resistance gene; Nh, Nhel; Ec, EcoRl; Xb, Xbal; Hi, Hindlll; 47, Eco47III; Ps, Pstl; Ns, Nsil; Nd, Ndel; RV, EcoRV; Bs, BspEl; Bg, Bg/ll; Pm, Pmel; Sm, Smal.

lividans and gene fusion of $l k c F$ and $l k c G$, the results of which are described in this paper.

\section{Results and Discussion}

\section{Heterologous Expression of the lkc Cluster (lkcA-lkcO) in S. lividans}

Although extensive gene disruption experiments have delimited the lankacidin biosynthetic $(l k c)$ cluster in the range from $l k c A$ to $l k c O$ (Fig. 3A) [9, 10], we could not completely rule out the possibility that additional genes located on pSLA2-L or the chromosome might be involved. To exclude this possibility, heterologous expression of the $l k c$ cluster was carried out in S. lividans. For this purpose, the $l k c$ cluster was divided into two regions, $l k c A-l k c E$ and $l k c F-l k c O$, because whole cluster is too large to be incorporated in one vector. Consequently, the $l k c A-l k c E$ region was integrated into the chromosome of $S$. lividans, while the $l k c F-l k c O$ region was inserted into the E. coliStreptomyces shuttle vector pKAR1026-4, a pHGF7604based vector [11]. To express the divided clusters in $S$. lividans, we used the ActII-Orf4/PactI-PactIII expression system. ActII-Orf4 is a transcriptional activator that binds to the pathway-specific actI and actIII promoters (PactI and PactIII) and initiates actinorhodin synthesis in Streptomyces coelicolor [12]. This system has been used for the expression of various antibiotic biosynthetic genes due to its strong promoter activity $[11,13]$.

To construct the $l k c A-l k c E$ region, a $0.67-\mathrm{kb}$ DNA fragment containing the $5^{\prime}$ terminus of $l k c A$ (nt 35,881 35,217 of pSLA2-L) and multiple cloning sites was amplified by polymerase chain reaction (PCR) (Fig. 3B-I). Then, the actII-orf4/PactIII region was amplified by PCR and introduced into this fragment, and finally the $13-\mathrm{kb}$ NheI-PstI (nt 22,293 35,217) fragment carrying the rest part of $l k c A$ and the $l k c B-l k c E$ genes was inserted to give pKAR1047 on the integrative shuttle vector pSET152 [14]. pKAR1047 was transformed into S. lividans TK64 and finally gave strain KA53, in which the $l k c A-l k c E$ region was integrated into the chromosome at the $\phi \mathrm{C} 31 \mathrm{attP}$ site (Fig. 3B-III).

To construct the $l k c F-l k c O$ cassette, a $0.48-\mathrm{kb}$ PCR fragment containing the $5^{\prime}$ terminus of $l k c F$ and multiple cloning sites was obtained (Fig. 3B-II). Then, the $5.7-\mathrm{kb}$ PstI-NheI (nt 16,616 22,293) fragment, the 2.8-kb EcoRI- 


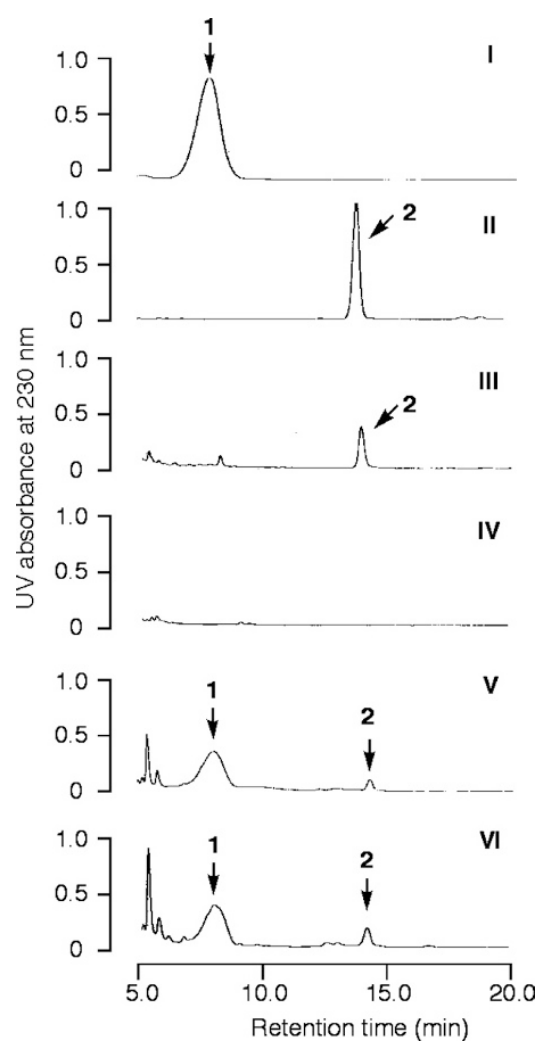

Fig. 4 Analysis of lankacidin production by reverse-phase HPLC.

(I) Lankacidin C (1), (II) lankacidinol A (2), (III) S. lividans KA53 (IkcA-IkcE) carrying pKAR1034 (IkcF-IkcO), (IV) KA53 (IkcA-IkcE) carrying pKAR1026-4 (control), (V) S. rochei 51252 (parent), and (VI) S. rochei KA49 (IKcFG fusant).

$B s p \mathrm{EI}$ (nt 4,659 7,450) fragment, and the 9.2-kb BspEIPstI (nt 7,450 16,616) fragment were successively introduced into this fragment. The obtained $18.2-\mathrm{kb}$ fragment containing whole $l k c F-l k c O$ region was cloned into pKAR1026-4 carrying actII-orf4 and PactI to give pKAR1034. This plasmid was transformed into $S$. lividans KA53 containing integrated $l k c A-l k c E$ genes.

Lankacidin production was analyzed by reverse-phase HPLC. S. lividans KA53 harboring pKAR1034 showed a peak of lankacidinol A (2) (Fig. 4-III), while strain KA53 harboring the control plasmid pKAR1026-4 did not (Fig. 4IV). The identity of the metabolite with 2 was confirmed by mass spectrometry $\left[(\mathrm{M}+\mathrm{Na})^{+}=526.1\right]$. The production level of 2 was relatively low $(0.40 \mathrm{mg} /$ liter $)$ compared with the parent strain $51252(8.0 \mathrm{mg} /$ liter of lankacidin C (1) and $0.25 \mathrm{mg} /$ liter of 2). However, this result clearly indicated that the gene cluster $(l k c A-l k c O)$ contains all genes necessary for the synthesis of the lankacidin skeleton, and ruled out a possibility that additional genes located on pSLA2-L or the chromosome are involved in this process.
Interestingly, $S$. lividans KA53/pKAR1034 did not produce $\mathbf{1}$ but produced only $\mathbf{2}$, although the former is a main product in strain 51252. $\mathbf{2}$ may be converted to $\mathbf{1}$ by subsequent oxidation of C-24 and hydrolysis of an acetoxy group on C-7 [10]. Therefore, additional enzymes necessary for these modifications may be coded on the Streptomyces rochei chromosome, but not on the S. lividans chromosome.

\section{lkcF-lkcG Fusant Produced Lankacidin at a Similar Level to the Parent Strain}

Heterologous expression of the $l k c$ cluster in S. lividans confirmed that eight condensation reactions for the lankacidin skeleton are accomplished by five KS domains in the cluster; one $\mathrm{KS}$ domain in LkcA, one in LkcC, two in $\mathrm{LkcF}$, and one in LkcG. This means that one (or some) of the four PKS proteins functions iteratively. LkcA is an NRPS-PKS fusion protein, recognizing a glycine starter unit and condensing it with a malonate unit. Therefore, LkcA could be eliminated from the candidate enzymes performing iterative condensation. Similarly, LkcG could be eliminated, because it contains a thioesterase (TE) domain and terminates chain elongation reaction. Thus, $\mathrm{LkcC}$ and $\mathrm{LkcF}$ remained as candidates by elimination.

Similar iterative use of modular PKSs was proposed for stigmatellin in Stigmatella aurantiaca [4], borrelidin in Streptomyces parvulus Tü4055 [5], and aureothin in Streptomyces thioluteus [6]. Based on the gene organization, it was suggested that either StiI or StiJ may be used twice for stigmatellin synthesis, BorA5 three times for borrelidin, and AurA twice for aureothin. However, any causative sequence differences between modular and iterative PKSs have not been recognized. Fusion of the iterative BorA5 protein with the neighbor PKSs (BorA4 or BorA6) did not abolish borrelidin production, but the production yield was greatly decreased (about $20 \%$ of the parent strain) [5]. Based on the former fact (positive production), Olano et al. [5] concluded that three copies of Bor5 were not used sequentially in borrelidin synthesis but one copy was used repeatedly. However, we rather focused on the latter fact (low production yield), because fusion of the typical modular PKSs, 6-deoxyerythronolide B synthases, did not affect the production yield of erythromycin [15]. Thus, it was suggested that fusion of iterative and modular PKSs decreases a production yield, which we utilized to distinguish an iterative PKS from modular PKSs in the following experiments.

To test an iterative function of LkcF, we constructed a gene fusant of $l k c F$ and $l k c G$. The $3^{\prime}$ terminus of $l k c F$ and the $5^{\prime}$ terminus of $l k c G$ were combined to make a fused $l k c F G$ gene, which codes a fused protein with the amino 
A

(I) Parent strain

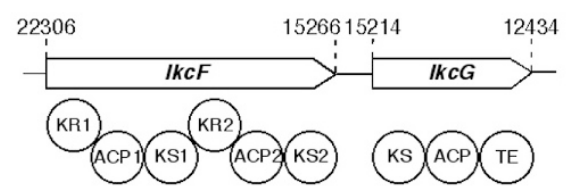

(II) IkcFG Fusant

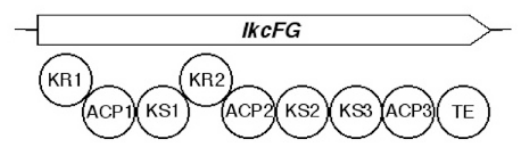

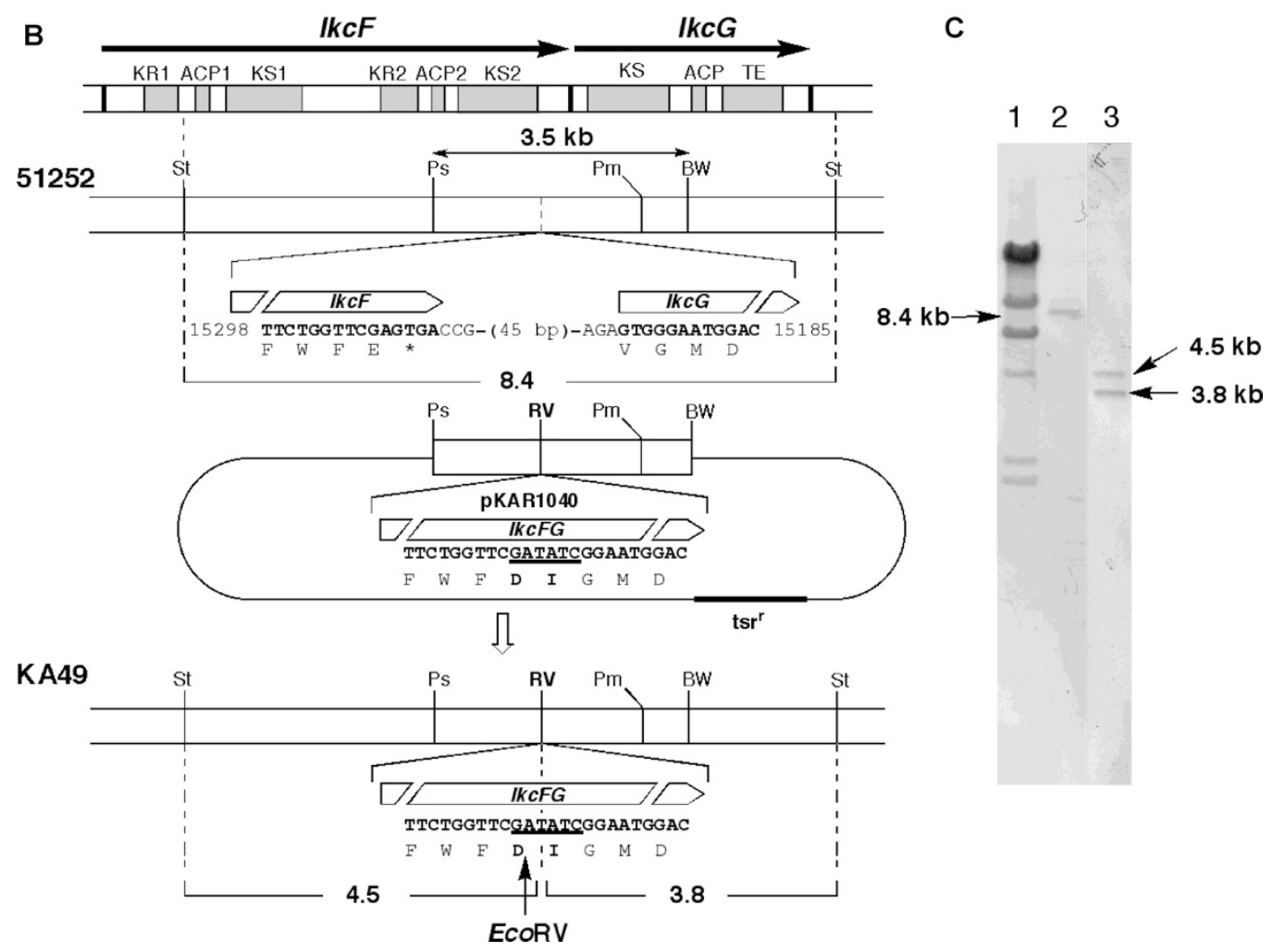

Fig. 5 Gene fusion of $I k c F$ and $I k c G$.

Domain organization of LkCF and LkcG in strain 51252 (I) and fusant KA49 (II). Numbers indicate the nucleotide positions in pSLA2-L. (B) Construction scheme of fusant KA49. The $N$ - and $C$-terminal amino acid sequences in 51252 and the fused sequence in KA49 are shown. An EcoRV site was generated in KA49. Ps, Pstl; St, Stul; BW, BsiVI; Pm, Pmel; RV, EcoRV. (C) Southern blot analysis. Lane 1, $\lambda /$ HindIII; lane 2, 51252/Stul-EcoRV; lane 3, KA49/Stul-EcoRV. Probe, 3.5-kb Pstl-BsiWI fragment in (B).

acid sequence FWFDIGMD in place of the $C$-terminal FWFE of LkcF and the $N$-terminal VGMD of LkcG (Figs. $5 \mathrm{~A}$ and B). Plasmid pKAR1040 containing the fused $l k c F G$ gene on the shuttle vector pRES18 [16] was transformed into Streptomyces rochei strain 51252, which finally gave mutant KA49 by gene replacement (Figs. 5B and C). As shown in Fig. 4-VI, fusant KA49 produced $\mathbf{1}$ and $\mathbf{2}$ at a similar level with the parent strain (Fig. 4-V). This result suggested that $\mathrm{LkcF}$ and $\mathrm{LkcG}$ are modular PKSs, leaving only $\mathrm{LkcC}$ as an iterative PKS by elimination. Although gene fusants of $l k c A-l k c C$ and $l k c C-l k c F$ are under construction, we have not yet obtained them due to the presence of intervening genes, $l k c B$ between $l k c A$ and $l k c C$, and $l k c D$ and $l k c E$ between $l k c C$ and $l k c F$.

\section{What Does Determine Modular or Iterative Condensation?}

The Lkc-PKS proteins, especially $\mathrm{LkcC}$, have several interesting features, which distinguish them from usual type-I PKS proteins. They do not contain AT or DH domains, both of which are present as discrete proteins, LkcD or LkcB. The domain order (KR-ACP-KS) of $\mathrm{LkcC}$ and $\mathrm{LkcF}$ is different from those (KS-ATKR-ACP) of usual type-I PKSs such as DEBS1 for 
erythromycin synthesis; namely, the KS domain is located at the $C$-terminus of protein in place of the $N$-terminal position. In addition, $\mathrm{LkcC}$ contains two tandemly aligned ACP domains. Are these properties related to iterative condensation reaction?

Different from the Lkc proteins, the PKS proteins for stigmatellin, borrelidin and aureothin biosynthesis contain AT and DH domains in normal domain arrangement. Thus, the unique domain order and the discrete AT domain of the Lkc proteins may not be related to iterative function. However, they gave us an interesting question on the evolution of PKS genes. It was suggested that monofunctional type-II PKS genes have been fused into a multifunctional type-I PKS gene in the evolutional history [17]. Therefore, in the generation process of the $l k c-P K S$ genes, the KS gene was fused with other genes in a unique order, while the AT and DH genes were not fused and remained as separate genes. The tandem alignment of two ACP domains was also found in the albicidin PKS [18] and several fungal type-I PKSs, for example, WA for naphtopyrone [19, 20] and StcA for sterigmatocystin [21]. Huang et al. [18] proposed that the second ACP domain in the albicidin PKS serves as a waiting position for growing chains to facilitate iterative condensation, although no experimental evidence was provided. Fujii et al. [21] disrupted each of the two tandem ACP domains in WA and showed that either ACP alone was enough for naphtopyrone synthesis. It is noteworthy that tandemly aligned doublet and triplet ACP domains were found even in the modular PKSs for mupirocin in Pseudomonas fluorescens [22]. All of these results suggest no relationship between tandem ACPs and iterative condensation. However, disruption of tamdem ACPs in $\mathrm{LkcC}$ is in progress to get a final answer on their function in lankacidin synthesis.

Despite of accumulated data, little information has been obtained on the question how the PKS enzymes for lankacidin, stigmatellin, borrelidin, and aureothin could distinguish and perform modular and iterative condensations. Although we extensively introduced amino acid replacement into the active sites of three KR domains in the $l k c C$ and $l k c F$ genes, we have not isolated any metabolites to confirm our hypothesis (unpublished results). Such metabolites have neither been isolated for stigmatellin, borrelidin, or aureothin. A new strategy with a totally different aspect may be necessary to obtain conclusive evidence.

In conclusion, heterologous expression of the lankacidin cluster and gene fusion of $l k c F$ and $l k c G$ in this study suggested a plausible hypothesis that $\mathrm{LkcC}$ is used four times and LkcA, LkcF and LkcG are used modularly to accomplish eight condensation reactions leading to the lankacidin skeleton.

\section{Experimental}

\section{Bacterial Strains and Culture Conditions}

Streptomyces rochei strain 51252 [7] carrying only pSLA2$\mathrm{L}$ was used as the parent strain of lankacidin producer. All of the strains, plasmids, and PCR primers used in this study are listed in Table 1. DNA manipulations for Escherichia coli [23] and Streptomyces [24] were performed according to the standard procedures. Streptomyces strains were grown in YEME medium [24], treated with lysozyme to make protoplasts, regenerated on R1M medium [25], and overlaid with soft nutrient agar containing thiostrepton (final concentration; $10 \mu \mathrm{g} / \mathrm{ml})$ or apramycin $(50 \mu \mathrm{g} / \mathrm{ml})$ to select transformants. YM medium (yeast extract $0.4 \%$, malt extract $1.0 \%$, and glucose $0.4 \%$, pH 7.3 ) was used for antibiotic production. Escherichia coli XL1-blue was grown in Luria Bertani medium containing either ampicillin $(100 \mu \mathrm{g} / \mathrm{ml})$ or apramycin $(50 \mu \mathrm{g} / \mathrm{ml})$.

\section{Heterologous Expression of the lkc Cluster (lkcA-lkcO)} Introduction of $l k c A-l k c E$ Genes into the Chromosome of $S$. lividans TK64

Using cosmid B10 of pSLA2-L [9] as a template and primers KAR-1801 and KAR-1802 (Table 1), a 0.67-kb DNA fragment containing the $N$-terminus of $l k c A$ (nt 35,881 35,217 of pSLA2-L) and multiple cloning sites was amplified. After treatment with NsiI-XbaI, this fragment was cloned into pKAR1024 to give pKAR1027. To introduce the PactIII promoter and an EcoRV site, another PCR product was prepared using pKAR1027 as a template and primers KAR-1802 and KAR-1803. The resulting DNA cut with $P a c \mathrm{I}-\mathrm{Xba \textrm {I }}$ was cloned into pNEB193 (New England Biolabs) to afford pKAR1044. Its vector fragment digested with $S m a \mathrm{I}-\mathrm{Xba \textrm {I }}$ was replaced by pBluescript SK-plus (Stratagene) digested with EcoRV$X b a \mathrm{I}$ to give pKAR1045. A 13-kb NheI-PstI fragment (nt $22,293 \sim 35,217$ ) was introduced into the corresponding sites of pKAR1045 to give pKAR1046, the vector part of which was replaced by pSET152 [14] digested with EcoRV$X b a \mathrm{I}$ to afford an integrative $l k c A-l k c E$ expression plasmid, pKAR1047. This plasmid was transformed into S. lividans TK64 and transformants were cultured in the presence of apramycin $(20 \mu \mathrm{g} / \mathrm{ml})$ to give strain KA53, in which the $l k c A-l k c E$ genes were integrated into the chromosome.

Construction of $l k c F-l k c O$ Expression Plasmid

Using cosmid B10 as a template and primers KAR-1301 and KAR-1303, a 0.48-kb fragment was amplified. After 
Table 1 Bacterial strains, plasmids, and primers used in this study

\begin{tabular}{|c|c|c|}
\hline Strains/plasmids/primers & Properties/oligonucleotide sequences & Source/ref. \\
\hline \multicolumn{3}{|l|}{ Strains } \\
\hline S. rochei 7434AN4 & Wild type (pSLA2-L, M, S) & Ref. 7 \\
\hline S. rochei 51252 & Strain from 7434AN4 (pSLA2-L) & Ref. 7 \\
\hline S. rochei KA49 & in-frame gene fusion of $I k c F$ and $I k c G$ & This study \\
\hline S. lividans KA53 & Strain TK64 with integration of pKAR1047, aac3(IV) & This study \\
\hline \multicolumn{3}{|l|}{ Plasmids } \\
\hline pHGF7505 & pNEB193-based plasmid carrying actII-orf4 and PactIII-actl & Ref. 11 \\
\hline pHGF7604 & SCP2*-derived vector, bla, tsr & Ref. 11 \\
\hline cosmid B10 & 45.4-kb pSLA2-L DNA (nt 3,341-48,756) cloned into SuperCos-1 at BamHI site & Ref. 9 \\
\hline pKAR1024 & 1.2-kb Swal-Pmel fragment eliminated from pHGF7505 & This study \\
\hline pKAR1025 & EcoRI site eliminated from pKAR1024 & This study \\
\hline pKAR1026 & 1.5-kb EcoRI-HindIII fragment carrying actII-orf4 and PactIII-act/ cloned into pHGF7604 & This study \\
\hline pKAR1026-4 & 1.5-kb EcoRI-Bg/ll PCR product cloned into pKAR1026 & This study \\
\hline pKAR1027 & 0.67-bp Nsil-Xbal PCR product cloned into pNEB193 & This study \\
\hline pKAR1029 & 0.48-bp Pacl-Smal PCR product cloned into pKAR1025 & This study \\
\hline pKAR1030 & 5.2-kb Nhel-Pstl fragment (nt 22,293 16,616) cloned into pKAR1029 & This study \\
\hline pKAR1032 & 2.8-kb BspEl-EcoRl fragment (nt 7,450 4,659) cloned into pKAR1030 & This study \\
\hline pKAR1033 & 9.2-kb Pstl-BspEl fragment (nt 16,616 7,450) cloned into pHGF1032 & This study \\
\hline pKAR1034 & 18-kb Pacl-Smal fragment cloned into pKAR1026-4 at Pacl-Pmel site & This study \\
\hline pKAR1044 & 0.67-bp Pacl-Xbal PCR product cloned into pNEB193 & This study \\
\hline pKAR1045 & 0.67-bp Smal-Xbal fragment from pKAR1044 cloned into pBluescript SK + at EcoRV-Xbal site & This study \\
\hline pKAR1046 & 13-kb Pstl-Nhel fragment (nt 35,217 22,293) cloned into pKAR1045 & This study \\
\hline pKAR1047 & 14-kb EcoRV-Xbal fragment from pKAR1046 cloned into pSET152 & This study \\
\hline pKAR1038 & 3.5-kb BsMl-Pstl fragment cloned into Acc65I-Pstl site of Litmus28i & This study \\
\hline pKAR1039 & 1.3-kb Agel-BbrPI fragment from pKAR1038 replaced with PCR fragment & This study \\
\hline pKAR1040 & 3.5-kb Stul-Pstl fragment from pKAR1039 cloned into pRES18 at Smal-Pstl region & This study \\
\hline \multicolumn{3}{|l|}{ Primers } \\
\hline KAR-1301 & 5'-GTGCTTAATTAACACGGGGATGACCCATGG-3' & This study \\
\hline KAR-1303 & 5'-AACCCGGGAATTCAGTCCGGACTGCAGCATCGATCCAC-3' & This study \\
\hline KAR7505f1 & 5'-GGCAGATCTCGATTATAACAGCTCGGATTC-3' & This study \\
\hline KAR7505r1 & 5'-GTTGAATTCGTTTAAACGAGCTCGGTACCC-3' & This study \\
\hline KAR-1801 & 5'-CAGAAATGCATATGACGGAGCGTCAGGCAA-3' & This study \\
\hline KAR-1802 & 5'-GATCTAGAGCTAGCGAACTGCAGCAGGCTC-3' & This study \\
\hline KAR-1803 & 5'-GCCTTAATTAAGATATCGCCGGATCCACCG-3' & This study \\
\hline KAR1312link02 & 5'-GCGAATTCACGTGTCCGTTGGAGC-3' & This study \\
\hline KAR1312link03 & 5'-GAACCGGTTCTGGTTCGATATCGGAATGGA-3' & This study \\
\hline
\end{tabular}

digestion with PacI-SmaI, this fragment was cloned into pKAR1025 to give pKAR1029. The 5.2-kb Pst I-NheI fragment (nt 16,616 22,293) was cloned into pKAR1029 to give pKAR1030, the 2.8-kb EcoRI-BspEI fragment (nt 4,659 7,450) was introduced into pKAR1030 to afford pKAR1032, and then the 9.2-kb BspEI-PstI fragment (nt $7,450 \sim 16,616)$ was introduced into pKAR1032 to give pKAR1033. The vector part of pKAR1033 digested with PacI-SmaI was replaced by pKAR1026-4 digested with PacI-PmeI to afford pKAR1034. Plasmid pKAR1026-4 was constructed as follows. The insert of pHGF7604 [11] was replaced by the $1.5-\mathrm{kb}$ EcoRI-HindIII fragment of pHGF7505 [11] to give pKAR1026, and its cloning sites were modified using primers KAR7505f1 and KAR7505r1 to give pKAR1026-4.

\section{In-frame Gene Fusion of $\boldsymbol{l k c F}$ and $\boldsymbol{l k c} \boldsymbol{G}$}

Primers KAR1312link02 and KAR1312link03 were designed to obtain a fused $l k c F G$ sequence (Fig. 5). Using these primers and cosmid $\mathrm{B} 10$ as a template, a $1.3-\mathrm{kb}$ PCR product was obtained. After digestion with AgeI-BbrPI, this fragment was substituted for the original AgeI-BbrPI 
fragment of pKAR1038 to give pKAR1039. The vector region of pKAR1038 was replaced by pRES18 to afford pKAR1040. This plasmid was transformed into strain 51252, plasmid-integrated strains were selected by thiostrepton $(10 \mu \mathrm{g} / \mathrm{ml})$, and thiostrepton-sensitive double-crossovered strains were identified by Southern hybridization to give the $l k c F G$-fusant KA49 (Fig. 5C).

\section{Isolation and Detection of Metabolites}

Three-days culture broth was extracted with EtOAc and analyzed by TLC, HPLC, and electrospray ionization mass spectrometry (ESI-MS). HPLC analysis was carried out on a COSMOSIL $5 \mathrm{C}_{18}$-MS-II column $(4.6 \times 250 \mathrm{~mm}$, Nacalai Tesque, Japan), which was eluted with a $3: 7$ mixture of acetonitrile and $10 \mathrm{mM}$ sodium phosphate buffer $(\mathrm{pH} 8.2)$ at a flow rate of $1.0 \mathrm{ml} /$ minute. $\mathbf{1}$ and $\mathbf{2}$ were detected at 8.5 and 14.8 minutes, respectively, by a Jasco MD-2010 multiwavelength photodiode array detector. Production yields of $\mathbf{1}$ and $\mathbf{2}$ were determined from their peak intensities on HPLC chromatogram using authentic samples. ESI-MS spectra were obtained by an ALLIANCE2690/ ZQ2000 mass spectrometer (Waters, USA).

Acknowledgments We thank Prof. Dr. Heinz G. Floss (University of Washington) and Dr. Tin-Wein Yu (Louisiana State University) for plasmids, pHGF7505 and pHGF7604. This work was supported by Grant-in-Aid for Scientific Research from the Ministry of Education, Culture, Sports, Science and Technology of Japan.

\section{References}

1. Staunton J, Weissman KJ. Polyketide biosynthesis: a millennium review. Nat Prod Rep 18: 380-416 (2001)

2. Wilkinson B, Foster G, Rudd BAM, Taylor NL, Blackaby AP, Sidebottom PJ, Cooper DJ, Dawson MJ, Buss AD, Gaisser S, Böhm IU, Rowe CJ, Cortés J, Leadlay PF, Staunton J. Novel octaketide macrolides related to 6deoxyerythronolide $\mathrm{B}$ provide evidence for iterative operation of the erythromycin polyketide synthase. Chem Biol 7: 111-117 (2000)

3. Hardt IH, Steinmetz H, Gerth K, Sasse F, Reichenbach H, Höfle G. New natural epothilones from Sorangium cellulosum, strains So ce90/B2 and So ce90/D13: Isolation, structure elucidation, and SAR studies. J Nat Prod 64: 847-856 (2001)

4. Gaitatzis N, Silakowski B, Kunze B, Nordsiek G, Blöker H, Höfle G, Müller R. The biosynthesis of the aromatic myxobacterial electron transport inhibitor stigmatellin is directed by a novel type of modular polyketide synthase. J Biol Chem 277: 13082-13090 (2002)

5. Olano C, Wilkinson B, Moss SJ, Sheridan R, Braña AF,
Méndez C, Leadlay PF, Salas JA. Evidence from engineered gene fusions for the repeated use of a module in a modular polyketide synthase. Chem Commun: 2780-2782 (2003)

6. He J, Hertweck C. Functional analysis of the aureothin iterative type I polyketide synthase. ChemBioChem 6: 908-912 (2005)

7. Kinashi H, Mori E, Hatani A, Nimi O. Isolation and characterization of large linear plasmids from lankacidinproducing Streptomyces species. J Antibiot 47: 1447-1455 (1994)

8. Kinashi H, Fujii S, Hatani A, Kurokawa T, Shinkawa H. Physical mapping of the linear plasmid pSLA2-L and localization of the eryAI and actI homologs. Biosci Biotech Biochem 62: 1892-1897 (1998)

9. Mochizuki S, Hiratsu K, Suwa M, Ishii T, Sugino F, Yamada $\mathrm{K}$, Kinashi H. The large linear plasmid pSLA2-L of Streptomyces rochei has an unusually condensed gene organization for secondary metabolism. Mol Microbiol 48: 1501-1510 (2003)

10. Arakawa K, Sugino F, Kodama K, Ishii T, Kinashi H. Cyclization mechanism for the synthesis of macrocyclic antibiotic lankacidin in Streptomyces rochei. Chem Biol 12: 249-256 (2005)

11. Yu TW, Müller R, Müller M, Zhang X, Draeger G, Kim CG, Leistner E, Floss HG. Mutational analysis and reconstituted expression of the biosynthetic genes involved in the formation of 3-amino-5-hydroxybenzoic acid, the starter unit of rifamycin biosynthesis in Amycolatopsis mediterranei S699. J Biol Chem 276: 12546-12555 (2001)

12. Fernández-Moreno MA, Caballero J, Hopwood DA, Malpartida F. The act cluster contains regulatory and antibiotic export genes, direct targets for translational control by the bldA tRNA gene of Streptomyces. Cell 66: 769-780 (1991)

13. McDaniel R, Ebert-Khosla S, Hopwood DA, Khosla C. Engineered biosynthesis of novel polyketides. Science 262: 1546-1550 (1993)

14. Bierman M, Logan R, O’Brien K, Seno ET, Rao RN, Schoner BE. Plasmid cloning vectors for the conjugal transfer of DNA from Escherichia coli to Streptomyces spp. Gene 116: 43-49 (1992)

15. McDaniel R, Kao CM, Hwang SJ, Khosla C. Engineered intermodular and intramodular polyketide synthase fusions. Chem Biol 4: 667-674 (1997)

16. Ishikawa J, Niino Y, Hotta K. Construction of pRES18 and pRES19, Streptomyces-Escherichia coli shuttle vectors carrying multiple cloning sites. FEMS Microbiol Lett 145: 113-116 (1996)

17. Hopwood DA. Genetic contributions to understanding polyketide synthases. Chem Rev 97: 2465-2497 (1997)

18. Huang G, Zhang L, Birch RG. A multifunctional polyketidepeptide synthetase essential for albicidin biosynthesis in Xanthomonas albilineans. Microbiology 147: 631-642 (2001)

19. Mayorga ME, Timberlake WE. The developmentally 
regulated Aspergillus nidulans $w A$ gene encodes a polypeptide homologous to polyketide and fatty acid synthases. Mol Gen Genet 235: 205-212 (1992)

20. Fujii I, Watanabe A, Sankawa U, Ebizuka Y. Identification of Claisen cyclase domain in fungal polyketide synthase WA, a naphthopyrone synthase of Aspergillus nidulans. Chem Biol 8: 189-197 (2001)

21. $\mathrm{Yu}$ JH, Leonard TJ. Sterigmatocystin biosynthesis in Aspergillus nidulans requires a novel type I polyketide synthase. J Bacteriol 177: 4792-4800 (1995)

22. Rahman AS, Hothersall J, Crosby J, Simpson TJ, Thomas CM. Tandem duplicated acyl carrier proteins, which increase polyketide antibiotic production, can apparently function either in parallel or in series. J Biol Chem 280: 6399-6408 (2005)

23. Sambrook J, Fritsch EF, Maniatis T. In Molecular cloning: A laboratory manual, Cold Spring Harbor Laboratory Press, Cold Spring Harbor, NY, USA (1989)

24. Kieser T, Bibb MJ, Buttner MJ, Chater KF, Hopwood DA, In Practical Streptomyces genetics, The John Innes Foundation, Norwich, United Kingdom (2000)

25. Zhang H, Shinkawa H, Ishikawa J, Kinashi H, Nimi O. Improvement of transformation system in Streptomyces using a modified regeneration medium. J Ferment Bioeng 83: 217-221 (1997) 